Proc. Indian Acad. Sci. (Earth Planet. Sci.), Vol. 91, No. 1, March 1982, pp. 85-94. (C) Printed in India.

\title{
Attenuation of electrons flux penetrating through the lower ionosphere
}

\author{
P N KHOSA, R R RAUSARIA and K L MOZA* \\ Physics Department, Regional Engineering College, Srinagar, India \\ *Department of Physics, S P College, Srinagar, India
}

MS received 14 June 1981; revised 1 December 1981

\begin{abstract}
The angular variation of elastic and inelastic scattering cross-sections has been calculated and used to study the energy deposition by precipitating electrons with the help of Monte Carlo Method. Monoenergetic, power law electron spectrum with isotropic and monodinational incidence starting at an altitude of $300 \mathrm{~km}$ have been used to obtain the angular and energy distributions at certain height intervals. In these calculations constant magnetic field has been used.
\end{abstract}

Keywords. Electron flux; ionosphere.

\section{Introduction}

The penetration of test charges, their attenuation and energy deposition in the ionosphere have been extensively studied (Spencer 1959; Chamberlain 1961, Rees 1963, 1969, 1970; Maeda 1963, 1965; Kamiyama 1966: Prasad and Singh 1972). The detailed study of changes in the angular distribution and corresponding energy attenuation of electron influx penetrating the ionosphere is scantly documented. More than half of the studies referred to above have considered the penetration of a monoenergetic flux of electrons using Bethe's average attenuation formula. In this paper an attempt has been made to study the role of the energy spectrum on the angular distribution of scattered particles and on the energy attenuation.

The study of the penetration of charged particles into the ionosphere depends on the nature of the scattering process which determines the trajectory and energy loss as the charged particles traverse through the ionosphere. The inelastic scattering cross-section determines the energy loss and the elastic scattering cross-section depicts the deflection of the incident particle. With this in view, we have computed the elastic and inelastic scattering cross-sections using Hartree-Fock atomic model (Rausaria et al 1973) so far neglected in Monte Carlo calculations (Maehlum and Stadsnes 1967; Wedde 1971; Berger et al 1970, 1974). Using these cross-sections, CIRA model atmosphere and a given incident flux the Monte Carlo calculation are carried out to obtain information about the angular variation of the flux into the atmosphere. It is found that isotropic distribution incident on the top of the ionosphere becomes anisotropic due to collisions in the lower ionosphere. This gets reflected in angular distribution of bremsstrahlung radiation. 


\section{Electron energy spectrom}

The experimental measurements have shown that the penetrating electron influx has a certain energy spectrum consistent with various measurements (O'Brien and Resoner 1971; Chase 1970). We assume that the distribution of electrons has a power law energy spectrum.

$$
\psi(E)=(E)^{-\gamma}
$$

where $\gamma$ is a factor which changes from one event to another and with the energy of the precipitating particles. Another form of the energy spectrum which finds experimental support (Rees 1969; Hoffman 1969) is the exponential distribution expressed as

$$
\psi(E)=\exp \left(-E / E_{0}\right)
$$

where $E_{0}$ is called the e-folding energy. With the assumed spectral distribution of electrons, the directional differential flux of electrons is expressed as

$$
i(\theta, E) \mathrm{d} E=K^{\prime} \psi(E) \mathrm{d} E,
$$

where $K^{\prime}$ is a constant and changes from event to event. In the present calculation the power law spectrum in the energy range $20 \mathrm{keV}$ to $200 \mathrm{keV}$ is taken to be of the form $2.4 \times 10^{8} E^{-4}$ electrons $\mathrm{cm}^{-2} \mathrm{~S}^{-1} \mathrm{Sr}^{-1} \mathrm{keV}^{-1}$. In addition, we have considered monoenergetic electrons of energy $20 \mathrm{keV}$. The angular distribution of the incident electron flux is assumed to be isotropic. An isotropic flux is one for which the number of incoming particles depend only on the size of the solid angle of acceptance and is independent of the direction of incidence.

\section{Penetration of energetic electrons}

Each electron is characterized by its momentum vector $\mathbf{K}=K(\sin \theta \cos \theta, \sin \theta$ $\sin \phi, \cos \theta$ ), and its altitude $h$ in the atmosphere. Instead of $h$ it is more convenient to use the atom column density above $h(n=$ atom density)

$$
N(h)=\int_{h}^{\infty} n\left(h^{\prime}\right) \mathrm{d} h^{\prime}
$$

Consider an electron after the $j$ th scattering at a column density $N_{j}$ with momentum vector $\mathbf{K}_{j}$ and angles $\theta_{j,} \phi_{j}$. We first calculate the column density

$$
N_{J+1}=N_{s}+\delta N \cos \theta_{j}
$$


where the next scattering event occurs. The column density traversed between events is obtained from the solution of

$$
R_{N}=\int_{0}^{\delta N} \exp \left(-S^{\prime} \sigma\right) \mathrm{d} S^{\prime}
$$

where $R_{N}$ is a random number between zero and one. Column density is generated using total scattering cross-section. The scattering process has been characterised by two angles; the azimuth angle $\phi$ and the scattering angle $\theta_{s}$. The probability distribution for $\phi$ has been taken to be random between $0-2 \pi$, and for $\theta_{s}$ we have used

$$
y D\left(\theta_{s}, E\right)=\frac{\int_{0}^{\theta_{s}} \mathrm{~d} \sigma / \mathrm{d} \Omega}{\int_{0}^{\pi} \mathrm{d} \sigma / \mathrm{d} \Omega} .
$$

Using the theorem on addition of spherical harmonics, the new angle of incidence is obtained from

$$
\cos \theta_{J+1}=\cos \theta_{J} \cos \theta_{s}+\sin \theta_{J} \sin \theta_{s} \cos \phi_{\text {. }}
$$

We have used Bethe's energy loss formula (Maeda 1965; Wedde 1971) to study the electron energy loss in continuous slowing down approximation

$$
\begin{aligned}
& -\frac{\mathrm{d} E}{\mathrm{~d} h}=2 \pi r_{0}^{2} Z \frac{N_{0}}{A} \frac{m_{0} c^{2}}{\beta^{2}}\left[\log \frac{m_{0} \nu^{2} E}{2 l^{2}\left(1-\beta^{2}\right)}-\left(2\left(1-\beta^{2}\right)^{1 / 2}-1+\beta^{2}\right) \ln 2\right. \\
& \quad+1-\beta^{2}+\frac{1}{8}\left(1-\left(1-\beta^{2}\right)^{1 / 2}\right]
\end{aligned}
$$

where $r_{0}=$ classical radius of electrons $\left(2.82 \times 10^{-13} \mathrm{~cm}\right)$,

$N_{0}=$ Avogadro's number $\left(6.02 \times 10^{23}\right)$,

$m_{0} c^{\mathrm{a}}=$ rest energy of electron $(511 \mathrm{keV})$,

$E=\frac{m v^{2}}{2}=$ electron kinetic energy,

$\boldsymbol{Z}=$ atomic number of air (7.3),

$A=$ atomic weight of air $(14 \cdot 2)$,

$I \quad$ = ionization potential (for air, we have taken $98 \mathrm{eV}$ as ionization potential value Wedde 1971)

and $\beta=v / c$ velocity of electrons in units of light velocity.

This equation has been solved and the computation has been carried out in terms of small height intervals. The dimension of (7) is $\mathrm{keV} \mathrm{cm}^{2} \mathrm{~g}^{-1}$. The energy between 1 and $200 \mathrm{keV}$ is divided into many small intervals. Using this equation the mean energy loss corresponding to the specified energy differences is converted into absorp- 
tion mass $\left(\mathrm{g} / \mathrm{cm}^{2}\right)$. The distance travelled by the particle is also in the units of absorption mass. Comparison of these two values gives the new energy of the particle at any desired height. In this way charged particles have been followed till they lose all their energy or penetrate through the region of interest.

\section{Results and discussion}

It was not possible to accommodate the large number of variables; smaller slabs and height intervals and thus the total memory core of the IBM-360 was utilized in running the program. A compromise of 20 height slabs, 20 energy intervals and 20 scattered angles are taken. The total number of particles followed in a given time depends on chosen energies and angles. The anisotropy of electron flux obtained by the detailed computation in the forward and backward directions is shown in figures 1,2 and 3. In these diagrams we have only depicted the nature of energy and angular distributions at certain height intervals. Figure (1) shows the angular distributions of the directional differential electron flux for a monoenergetic electron of $20 \mathrm{keV}$ as it penetrates into the ionosphere. The ionosphere is divided into small slabs and after crossing each slab the angular and energy distributions of incident electron flux change significantly. It is seen from figure 1 that the angular distribution of scattered electron flux becomes anisotropic around $200 \mathrm{~km}$. The electrons retain their anisotropic nature in their

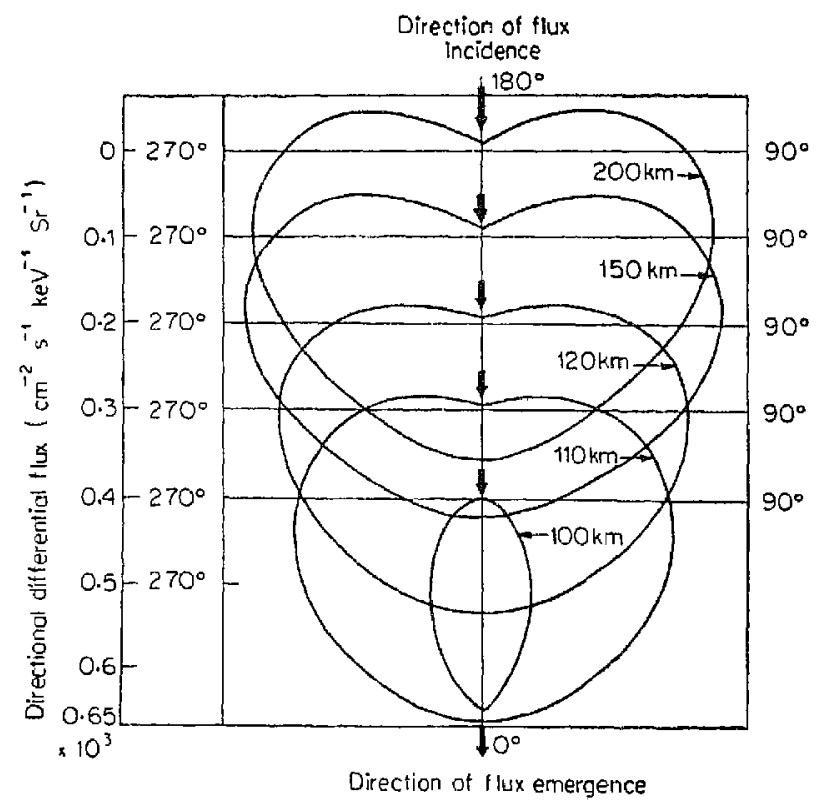

Figure 1. Angular distribution of the directional differential electron flux at different heights for a monoenergetic electron of $20 \mathrm{keV}$. 


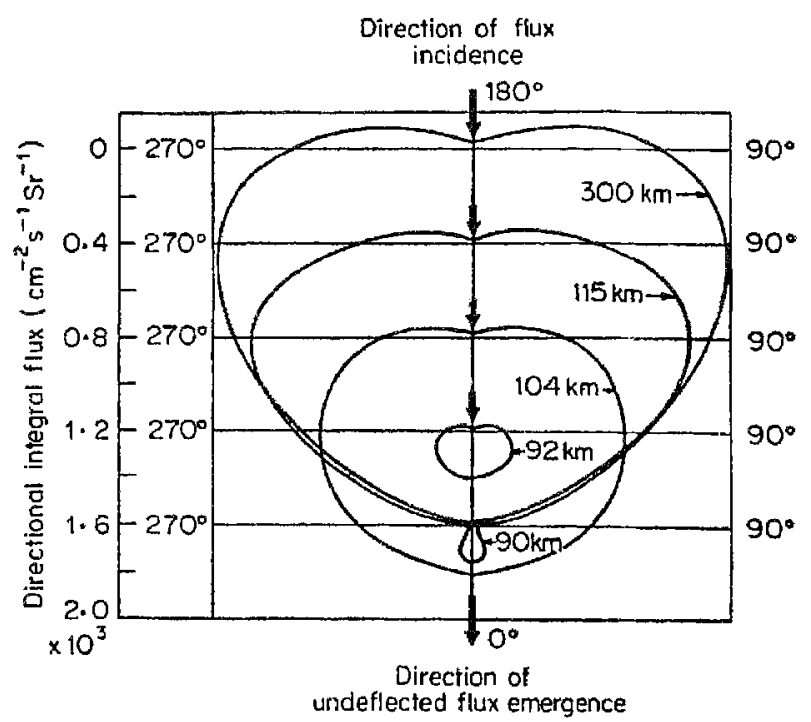

Figure 2. Angular distribution of directional integral electron flux corresponding to power law spectrum for energy greater than $20 \mathrm{keV}$ at different heights.

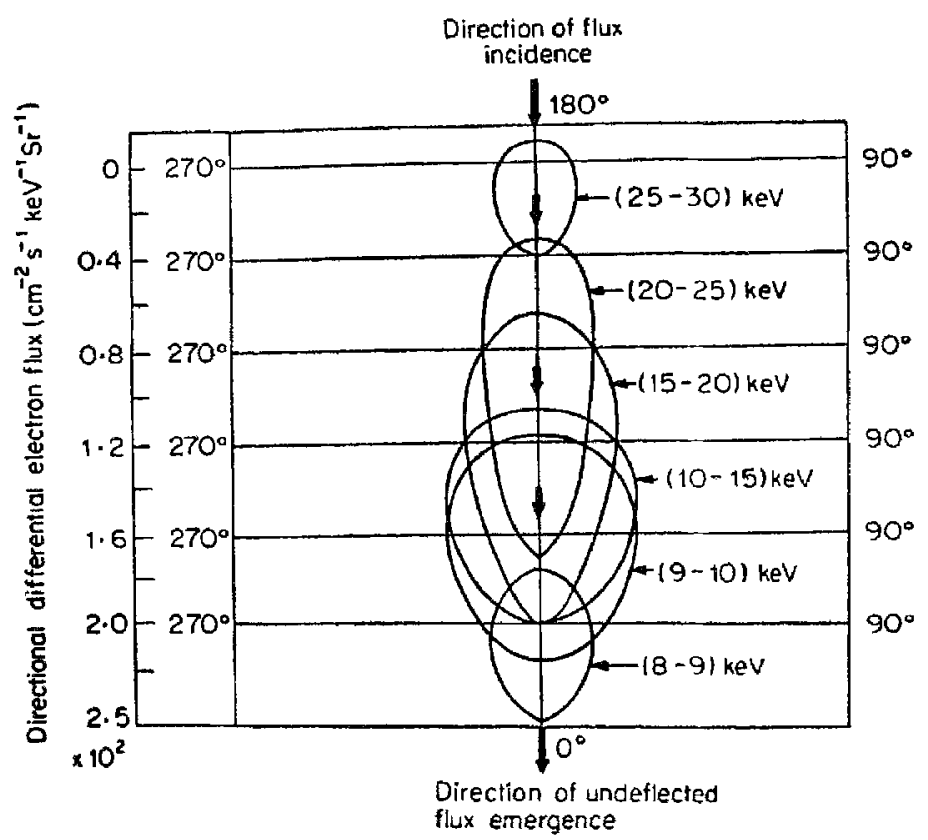

Figure 3. Angular distribution of directional differential electron flux for power law spectrum at fixed height $98 \mathrm{~km}$. 
downward journey. There is significant peaking in the distribution of scattered electrons at small angles due to minimum distance traversed. As the scattered angle increases, the distance traversed also increases and the particles are lost due to more collisions. This effect could be minimised by carrying out calculations taking very large numbers of thin slabs. Only electrons with smaller angles reach lower heights, therefore, the probability of back scattering decreases as the electron influx penetrates deeper and deeper in the ionosphere. Figure 2 shows the angular variation of the flux for energies greater than $20 \mathrm{keV}$ for an initially isotropic distribution with a power law spectrum. From the figure, it is seen that the angular distribution at $300 \mathrm{~km}$ is nearly isotropic. We find that the electron influx in the forward direction decreases rather faster (compared to figure 1) with penetrating depth. The sudden decrease in the electron flux is seen in the height range of $90-100 \mathrm{~km}$. The flux at $100 \mathrm{~km}$ becomes fairly anisotropic. The anisotropy of the scattered electron influx due to oblique incidence is almost the same for monoenergetic, power law and exponential electron energy spectra.

In figure 2 we have shown the integrated redistribution of scattered flux over the entire energy spectrum at different heights during its journey downwards. The redistribution of the scattered flux for various energies at a particular height is of interest. In figure 3 we have shown the angular variation of scattered electrons in the energy range (25-30), (20-25), (15-20), (10-15), (9-10) and (7-8) keV respectively at a fixed height $98 \mathrm{~km}$. The figure shows significant change in angular distributions between close energy intervals, at a particular height. These changes arise due to the energy and angular dependence of the scattering cross-section. This only reveals the detail of the intermediate process of the angular distribution. From this figure, we find that the magnitude of the scattered electron flux changes significantly in each energy range. The flux is seen to be more or less isotropic in the energy range (9-10) $\mathrm{keV}$. Figures 2 and 3 together give the complete information about the changes taking place in the scattered power law particle flux and their energy distribution. The detailed features depicted by these curves are consistent with the result reported by Rees (1969). Similar variation is also found with exponential electron spectrum.

A look at figures 1, 2 and 3 gives a good comparative idea of electron flux and energy distribution of scattering electrons. These figures depict only the intermediate process of flux and energy attenuation.

Scrutinizing these figures and cross comparing one notes the following:

(i) The angular distribution of scattered monoenergetic electron flux is less affected as compared to the scattered angular distribution of electrons obeying power law energy spectrum. The mono-energetic electron becomes anisotropic after travelling a large distance as compared to electrons following power law energy spectrum.

(ii) The back scattering coefficient is almost absent in the case of mono-energetic electrons at lower heights whereas small back scattered electrons are seen in the case of power law electron spectrum.

\subsection{Electron energy deposition and electron-ion pair production}

Rigorous calculations of energy attenuation considering monoenergetic charged particles were carried out by (Maeda 1965), Maehlum and Stadnes (1967) and Berger 
et al $(1970,1974)$. Using isotropic incidence of electrons, the exponential and power law energy spectra and the mean energy loss given by (7) we have computed the total energy deposition as a function of height. The total energy deposition height profile thus obtained has been shown in figure (4). In table 1, we have compared the energy loss of $20 \mathrm{keV}$ electrons with those of Berger et al (1974) and Wedde (1970). In contrast to Berger et al (1970) who have studied precipitation of low energy electrons $(2-20 \mathrm{keV})$ we have studied the passage of flux covering an extended energy range (20-200 keV). The compatibility and validity of our computation can however be checked from the comparison of theoretical and experimental electron-ion pair

Table 1. Energy loss rate for $20 \mathrm{keV}$ electron in units of $\mathrm{keV} / \mathrm{km}$

\begin{tabular}{|c|c|c|c|c|}
\hline \multirow{2}{*}{ Height } & \multicolumn{2}{|c|}{ Our calculated- $\mathrm{d} E / \mathrm{d} h$} & \multirow{2}{*}{$\begin{array}{c}\text { Berger et al's } \\
-\mathrm{d} E / \mathrm{d} h\end{array}$} & \multirow{2}{*}{$\begin{array}{l}\text { Wedde's } \\
-\mathrm{d} E / \mathrm{d} h\end{array}$} \\
\hline & With straggling & Without straggling & & \\
\hline 94 & $5.0(-1)$ & $7 \cdot 0(-2)$ & $5 \cdot 0(-1)$ & $9 \cdot 0-(1)$ \\
\hline 98 & $2 \cdot 5 \quad(0)$ & $1 \cdot 5 \quad(0)$ & $2.5 \quad(0)$ & $1.5 \quad(0)$ \\
\hline 102 & $1 \cdot 3 \quad(0)$ & $7.5(-1)$ & $1 \cdot 4$ & $6 \cdot 0(-1)$ \\
\hline 106 & $5.0(-1)$ & $4.0(-1)$ & $6.0(-1)$ & $2.5(-1)$ \\
\hline 110 & $2 \cdot 0(-1)$ & $1.9(-1)$ & $3.0(-1)$ & $9 \cdot 0(-2)$ \\
\hline 114 & $9 \cdot 0(-2)$ & $5.8(-2)$ & $1.5(-1)$ & $4 \cdot 0(-2)$ \\
\hline 118 & $3.5(-2)$ & $2 \cdot 3(-2)$ & $8 \cdot 0(-2)$ & $1.9(-2)$ \\
\hline 120 & $1.9(-2)$ & $1 \cdot 1 \quad(-2)$ & $7 \cdot 0(-2)$ & $1 \cdot 5(-2)$ \\
\hline
\end{tabular}

Values in parenthesis denote power of ten.

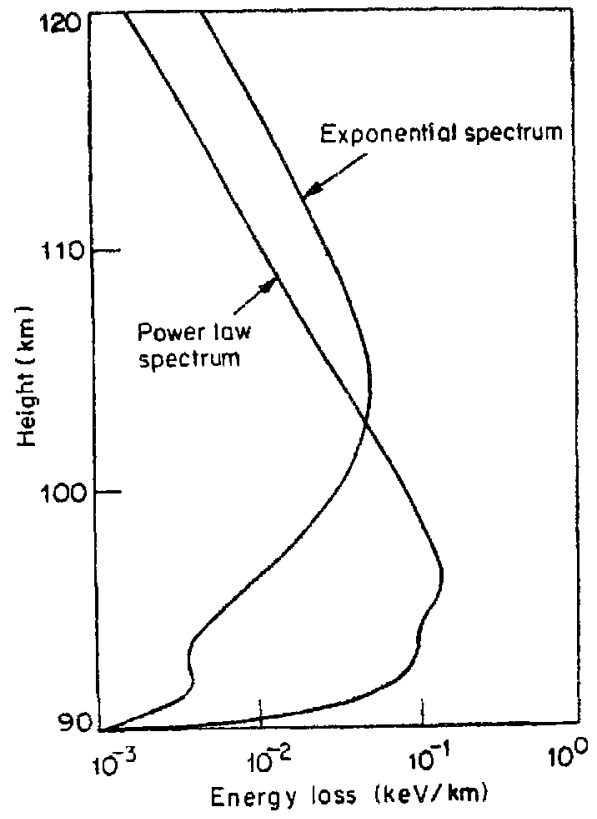

Figure 4. Energy loss profile of experimental and power law electron spectra. 
Table 2. Electron-ion pair production rate for $20 \mathrm{keV}$ monoenergetic electrons in units of $\mathrm{cm}^{-8} \mathrm{~s}^{-1}$

\begin{tabular}{ccccc}
\hline $\begin{array}{c}\text { Height } \\
\mathrm{km}\end{array}$ & Without straggling & With straggling & $\begin{array}{c}\text { Berger } \text { et al's } \\
-(1970) q\end{array}$ & $\begin{array}{c}\text { Wedde's (1970) } \\
q\end{array}$ \\
\hline 120 & $2.98(-6)^{*}$ & $4.12(-6)$ & $1.50(-5)$ & $3.50(-6)$ \\
115 & $1.20(-5)$ & $2.05(-5)$ & $3.10(-5)$ & $9 \cdot 00(-6)$ \\
110 & $5.19(-5)$ & $7.95(-5)$ & $7.80(-5)$ & $3.00(-5)$ \\
105 & $1.62(-5)$ & $2.07(-4)$ & $1.80(-4)$ & $8.00(-5)$ \\
100 & $2.61(-5)$ & $4.75(-4)$ & $4.60(-4)$ & $3.50(-4)$ \\
96 & $2.64(-5)$ & $7.13(-4)$ & $6.20(-4)$ & $4.50(-4)$ \\
94 & $2.19(-5)$ & $1.42(-4)$ & $1.50(-4)$ & $3.00(-4)$
\end{tabular}

This table is prepared from data given by Berger et al (1970) and Wedde (1970)

*Values in parenthesis denote power of ten.

production rates. From the scrutinity of figure 4, we find that the power law spectral attenuation has a more pronounced peak and penetrates deeper into atmosphere. The energy loss profile due to power law and exponential spectra both show tendency of double peak formation. In case of power law spectra the two peaks are separated by a few kilometres and these are of equal strength. However, in the case of exponential spectra a less pronounced peak appears at about $93 \mathrm{~km}$ and flat energy deposition maxima appear at $104 \mathrm{~km}$. The total energy deposited into the ionosphere due to attenuation of energetic particles produce impact ionisation and a part of this energy is spent in producing electromagnetic radiation. The electromagnetic radiations further interact with the ambient gas and produce secondary ionisation. The electron-ion pair production rate $q$ at a given height is controlled by total energy input into the layer. According to Maeda (1963), Kamiyama (1966) one electron-ion pair on an average is produced by the absorption of $32 \mathrm{eV}$ energy. Therefore, the ion production rate $q(E, h, \theta)$ due to the flux $i(E, h, \theta)$ is given by

$$
q(E, h, \theta)=1 / 32(-\mathrm{d} E / \mathrm{d} h) i(E, h, \theta)
$$

Using the height profile of energy deposition, we have computed the corresponding electron-ion pair rates at different heights. The ion production rates at different heights are shown in table 2 and compared with various available observed values in table 3. From table 3 it is clear that ion-pair production due to power law spectrum during night are close to observed values. This shows that electron precipitation may be an important source of ionisation during night time. 


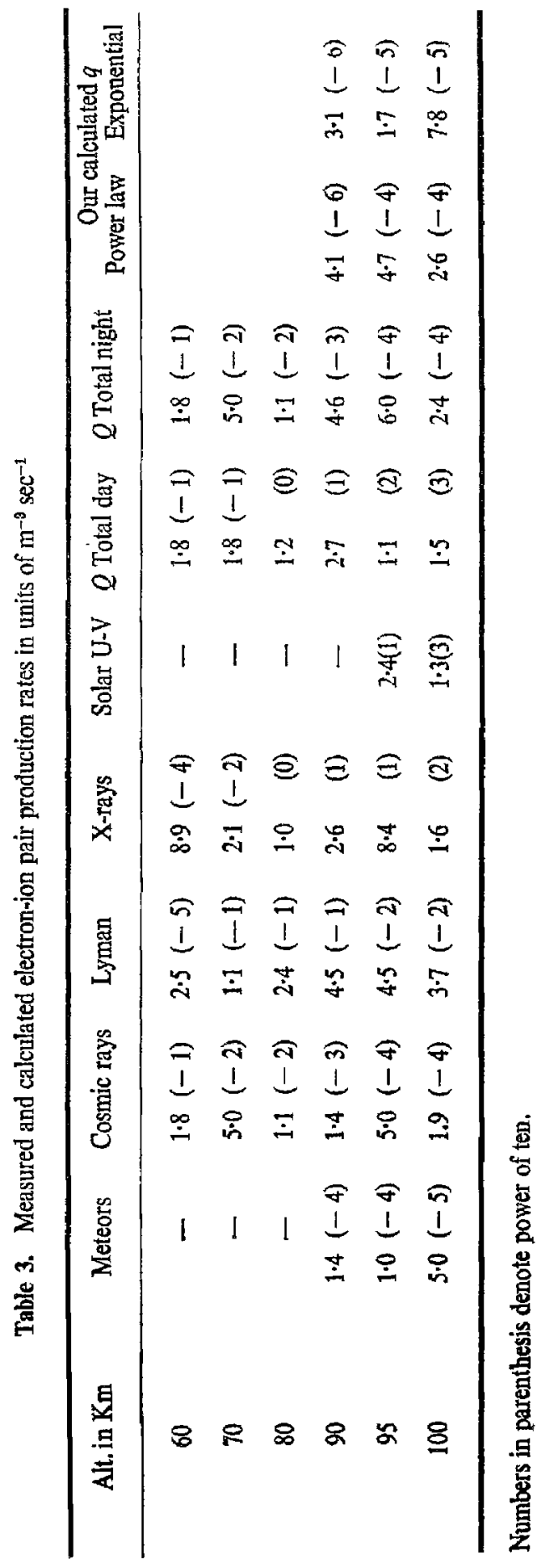




\section{Acknowledgement}

We are thankful to Dr O N Wakhloo for his encouragement and keen interest in the work.

\section{References}

Berger M J, Seltzer S M and Maede K 1970 J. Atmos. Terr. Phys. 321015

Berger M J, Seltzer S M and Maeda K 1974 J. Atmss. Terr. Phys. 36591

Chamberlain J W 1961 Physics of Aurora and Airglow (New York: Acndemic Press)

Chase L M 1970 J. Geophys. Res. 757128

Hoffman. R A $1969 \mathrm{~J}$. Geophys. Res. 742425

Kamiyama H 1966 Rep. Ionos. Space Res. Jpn. 20374

Maeda K 1965 Diffusion of Auroral Electrons in the Atmosphere NASA Tech. Note TND-2612

Machlum B and Stadsnes S 1967 Phys. Nory. 2111

Maeda K $1963 \mathrm{~J}$. Geophys. Res. 63185

O'Brien B J and Resone: D L $1971 \mathrm{~J}$. Geophys. Res. 768288

Prasad R Y and Singh R N 1972 Ann. Geoplys. 28555

Rausaria R R, Singhal R P and Singh R N 1973 Phys. Lett. A46 85

Rees M H 1963 Planet Space Sci. 21209

Rees M H 1969 Space Sci. Rev. 8413

Rees M H 1970 In *The polar ionospheric and magnetospheric processes (ed) G Skovli, (New York: Gordon and Breach)

Spencer L V 1959 Energy dissipation by fast electrons N B S Monograph No. 1 US Department of Commerce, National Bureau of Standards, Washington

Wedde T 1970 Intern. Rapport E-162, Notwegian Defence Research Establishment

Wedde T $1971 \mathrm{~J}$. Atmos. Terr. Phys. 33547 